\title{
Effectiveness and safety of insulin glargine Gla-300 in insulin- naïve type 2 diabetes subjects in a real-life setting -the GOAL_RO trial
}

\author{
Daniela Stegaru ${ }^{1}$, Simona Nicodim², Delia Vladu ${ }^{3}$, Olimpia Guțu ${ }^{4}$, Adriana Onaca ${ }^{5}$, Florina Pîrvu ${ }^{6}$, \\ Mihaela Moise $^{7}$, Cristian Guja ${ }^{1 \wedge}$; for the GOAL_RO Study Group
}

${ }^{1}$ Department of Diabetes, Nutrition and Metabolic Diseases, "Carol Davila” University of Medicine and Pharmacy, Bucharest, Romania; ${ }^{2}$ Emergency Clinical County Hospital Constanța, Constanța, Romania; ${ }^{3}$ Emergency Clinical County Hospital Craiova, Craiova, Romania; ${ }^{4}$ Olimpia Med SRL, Iași, Romania; ${ }^{5}$ Grandmed Medical Center, Oradea, Romania; ${ }^{6}$ Municipal Hospital “Dr. Gheorghe Marinescu” Târnăveni, Mureș, Romania; ${ }^{7}$ Sanofi Romania, București, Romani

Contributions: (I) Conception and design: C Guja, M Moise; (II) Administrative support: M Moise; (III) Provision of study materials or patients: All authors; (IV) Collection and assembly of data: All authors; (V) Data analysis and interpretation: C Guja, M Moise; (VI) Manuscript writing: All authors; (VII) Final approval of manuscript: All authors.

Correspondence to: Assoc Prof. Cristian Guja. 1st Clinic of Diabetes, Nutrition and Metabolic Diseases, "Carol Davila" University of Medicine and Pharmacy, Bucharest, 5-7 Ion Movila Street, Bucharest 2, Romania. Email: cristian.guja@b.astral.ro.

Background: Basal insulin is the first choice for insulin initiation in type 2 diabetes (T2DM), with the second generation of basal insulin analogues having a lower risk of hypoglycemia compared to the first generation of basal insulins. The aim of our study was to assess on a large cohort of insulin-naïve T2DM subjects the effectiveness and safety of insulin glargine $300 \mathrm{U} / \mathrm{mL}$ (Gla-300) in a real-life setting.

Methods: This was a multicenter, prospective, non-interventional, 24 weeks, 3 visits (baseline, 3 and 6 months) trial performed in adult T2DM subjects not achieving glycemic target (HbA1c $>7 \%)$ with prior oral or GLP-1 RA therapy. The study included 1,095 subjects (55.2\% M/44.8\% F) in 124 study sites. Mean $( \pm \mathrm{SD})$ age was $61.1 \pm 8.5$ years while mean duration of diabetes was $8.8 \pm 5.2$ years. Mean BMI was $31.7 \pm 5.4 \mathrm{~kg} / \mathrm{m}^{2}$ with $91.2 \%$ being overweight or obese. Baseline diabetes treatment included metformin (88.4\% of subjects), sulphonylureas (75.4\%), DPP-4i (16.7\%) and GLP-1 RAs (8\%). Comparison between quantitative variables was made with the paired sample $t$ test.

Results: Mean HbAlc at baseline was $9.8 \% \pm 1.7 \%$ with a mean fasting plasma glucose (FBG) of $231.5 \pm 67.4 \mathrm{mg} / \mathrm{dL}$. Mean HbA1c decreased to $7.7 \% \pm 1.2 \%$ at 6 months with a mean change from baseline of $-2.1 \%(\mathrm{P}<0.001)$. Overall, $30.7 \%$ of subjects reached the HbA1c target of $7 \%$. Final mean dose of Gla300 was $0.4 \mathrm{IU} / \mathrm{kg} /$ day. Mean weight gain was $0.4 \mathrm{~kg}$ over 6 months. Adverse events (AEs) were reported by $11.1 \%$ of subjects with $2.3 \%$ reporting serious adverse events (SAEs). Overall, $4.4 \%$ of subjects reporting at least one event of symptomatic or confirmed hypoglycemia. Only 7 episodes of nocturnal and one of severe hypoglycemia were reported.

Conclusions: In conclusion, a significant 2.1\% decrease of HbA1c was recorded after 6 months of treatment with Gla-300 with no unexpected safety signals, low risk of hypoglycemia and modest weight gain.

Keywords: Type 2 diabetes (T2DM); insulin treatment; insulin glargine Gla-300; real-life data

Submitted Jun 06, 2020. Accepted for publication Sep 30, 2020.

doi: $10.21037 /$ atm-20-4533

View this article at: http://dx.doi.org/10.21037/atm-20-4533

$\wedge$ ORCID: 0000-0002-8703-0522. 


\section{Introduction}

Despite apparently attenuating in developed countries, the type 2 diabetes (T2DM) epidemic continues to represent a major challenge worldwide, with approximately 463 million people affected in the 20-80 years old age group (1). In addition, T2DM is a major cause of morbidity and premature mortality $(\sim 11 \%$ of deaths in adults are attributable to diabetes) (1), with economic costs expected to rise to over 2 billion USD in the next decade (2). Most of these costs are related to the chronic complications of the disease that can be mostly prevented by achieving and maintaining glycemic targets, i.e., HbA1c below $7 \%$ $(53 \mathrm{mmol} / \mathrm{mol})$ for the majority of non-pregnant adults $(3,4)$.

Current guidelines of the American Diabetes Association's (ADA) and European Association for the Study of Diabetes (EASD) tend to recommend a glucagonlike peptide 1 receptor agonist (GLP-1 RA) as the first injectable medication in T2DM subjects due to potential $\mathrm{CV}$ benefits, associated weight loss and low risk of hypoglycemia $(3,4)$. However, reported adherence to GLP$1 \mathrm{RA}$ in real life observational studies is rather poor (5) and this leads to a $\sim 0.5 \%$ difference in HbA1c obtained in randomized controlled trials compared to real-world data (6). Approximately $75 \%$ of the gap seems to be explained by poor medication adherence.

On the other hand, insulin has the advantage of lowering blood glucose in a dose dependent manner and when appropriately titrated, it can decrease HbA1c up to almost any target $(3,4,7)$, limited only by the risk of hypoglycemia. In fact, due to the evolutive nature of the disease, many patients will need insulin therapy at some point during T2DM evolution in order to reach/maintain glycemic targets (8). Moreover, guidelines currently recommend early initiation of insulin in severely symptomatic patients or in the presence of high HbA1c/fasting blood glucose (FPG) $(3,4,7)$. However, insulin treatment initiation is quite often delayed due to multiple barriers, both related to physicians and patients (9). Proper patient education and their involvement in self-monitoring of blood glucose and self-titration of insulin dose may overcome some of the barriers and improve glucose control.

Basal insulin added to metformin, other oral drugs or a GLP-1RA is usually the first choice for insulin initiation $(3,4)$ due to lower risk of hypoglycemia and weight gain in comparison with prandial or premix insulins. The initial recommended dose is of 10 international units QD (or $0.2 \mathrm{IU} / \mathrm{kg} / \mathrm{day}$ ) with consequent insulin dose titration for attaining the target of FBG, usually set to $70-130 \mathrm{mg} / \mathrm{dL}$. Basal insulin analogs [glargine $100 \mathrm{U} / \mathrm{mL}$ (Gla-100), detemir] have a lower risk of hypoglycemia (mainly nocturnal) in comparison with NPH insulin while the second generation of basal insulin analogs [degludec and glargine $300 \mathrm{U} / \mathrm{mL}$ (Gla300)] exhibit an even lower risk compared to Gla-100 $(10,11)$.

Gla-300 is a second-generation basal insulin analog approved for clinical use since 2015 (12). It has the same molecular structure and metabolism but is $3 \times$ more concentrated in comparison with insulin Gla-100 (13). Consequently, the pharmacokinetics and pharmacodynamics of this insulin preparation are modified, with a prolonged duration of action (half-life of 19 hours and total duration beyond 24 hours), almost peak-less action profile and low intra-individual variability (14). These benefits were expected to translate in clinical benefits for diabetes subjects, both in T1DM and T2DM.

The efficacy and safety of Gla-300 was assessed in the large phase 3 EDITION clinical program of randomized controlled trials (RCTs) with Gla-100 as active comparator. The T2DM studies performed in Caucasians included the EDITION-1 (patients treated basal-bolus with NPH or Gla-100 as basal insulin), EDITION-2 (patients treated with basal insulin more than 42 IU QD and oral drugs) and EDITION-3 (patients treated only with oral drugs) (12). Primary efficacy and safety were evaluated after 6 months of treatment, with subsequent extension to 12 months. The meta-analysis of EDITION 1-3 trials indicated similar efficacy compared with insulin glargine (HbAlc decrease with $1.02 \%$ for both insulins and approximately $36 \%$ of subjects reaching $<7 \% \mathrm{HbA1c}$ target) (15). This was achieved with significant lower rates of confirmed/severe hypoglycemia for Gla-300 (RR 0.86, $\mathrm{P}=0.0116$ ), especially due to reduced number of nocturnal hypoglycemia. Severe hypoglycemia was rarely reported, with numerically fewer episodes for Gla-300 (RR 0.85, 95\% CI: 0.52 to 1.39) (15).

During the last decade, there was increasingly acceptance of the fact that results of randomized controlled trials (RCTs), usually including highly selected study populations, cannot be easily expanded to the heterogeneous population encountered in routine clinical practice. Consequently, there is an increasing demand from the main stakeholders in medicine for the use of results from real world evidence (RWE) studies in order to increase the robustness of data regarding the effectiveness and safety of different medicinal products (16).

In accordance to the higher emphasis placed recently on evidence provided by the RWE studies, the aim of our 
study was to assess in real life clinical practice in Romania the effectiveness and safety of insulin Gla-300 initiated in insulin-naïve T2DM subjects.

We present the following article in accordance with the TREND reporting checklist (available at http://dx.doi. org/10.21037/atm-20-4533).

\section{Methods}

The study was conducted in accordance with the Declaration of Helsinki (as revised in 2013) and the ICH-GCP regulations. Approval by institutional ethics committees was obtained for each participating site. All subjects provided written informed consent prior to any study related procedure. Data monitoring was provided by the sponsor.

\section{Design of study}

This was a multicenter, prospective, observational/noninterventional, open label, 24 weeks/3 visits [baseline (V1), 3 months (V2) and 6 months (V3)] trial. It was performed in 124 active study sites (outpatient departments of diabetes) from large/medium size cities distributed across Romania, between May 2017 and June 2018. Recruitment of study subjects was made by each investigator (selfselection). Insulin Gla-300 was initiated at the decision of each investigator and no restrictions on diabetes or other treatments and no interference with dosage regimens recommended by practicing physicians were imposed. Insulin Gla-300 was self-injected QD, sub-cutaneous by each study subject at home using the Solostar ${ }^{\circledR}$ injecting pen. There were no specific interventions to increase patient adherence.

\section{Inclusion/exclusion criteria}

Subjects eligible for inclusion in the trial were adult T2DM subjects (18-75 years), not achieving glycemic target (HbA1c $>7 \%$ ) with prior oral or GLP-1 RA therapy, insulin-naïve, initiated with insulin Gla-300 upon the decision of the prescribing physician and have signed the informed consent prior to any study procedure.

Subjects were excluded if having type 1 diabetes, contraindications to insulin treatment, previous insulin treatment for more than a week within one year prior to study inclusion, pregnancy or breastfeeding or intention to become pregnant during the duration the study, concomitant participation in another clinical trial, concomitance of severe medical conditions which might interfere with their participation in the study, severe mental conditions and refusal to sign the informed consent form.

\section{Study objectives}

The primary endpoint of the study was the change in HbA1c from the inclusion in the study up to the end of the follow-up period of 6 months.

Secondary objectives included percentage of subjects reaching HbA1c $\leq 7.0 \%$ after 6 months, percentage of subjects reaching the individualized target of HbA1c after 6 months, change from baseline in fasting plasma glucose (FPG) after 3 and 6 months, change from baseline in the dose of insulin Gla-300 after 3 and 6 months, change in body weight from baseline to 6 months and the frequency of confirmed/symptomatic, nocturnal or severe hypoglycemic episodes. The 2013 criteria of the ADA/Endocrine Society of hypoglycemia definition were used (17).

Other study objectives included stratification based on the initial value of HbA1c ( $<$ or $\geq 8 \%$ ) of the percentage of subjects reaching $\mathrm{HbA1c} \leq 7.0 \%$ after 6 months.

\section{Study assessments}

The data collected during each visit was related to those captured and documented during current care of each subject. The following was recorded: the demographical and anthropometric data of the patient (height, weight, waist circumference), the medical history (other than diabetes), duration of diabetes, presence of diabetes and cardiovascular complications, the individual glycemic targets proposed by physicians, concomitant medication (other than antidiabetics), the dose of insulin Gla-300 at all visits, the diabetes medication prior to entering the trial. Data were obtained from the medical records of the patient from the database of the treating physician according to the current practice. Blood samples were collected for the compulsory assessment of HbA1c and (optionally) of FPG at baseline and at 6 months.

Information was collected regarding the frequency with which physicians recommend a particular insulin titration algorithm and its description [collected data included target fasting blood glucose performed by patients using SMBG, frequency of dose adjustments (per week) number of insulin units for each step of titration, person who initiates titration (physician vs. patient) etc.]. Influence of the titration 


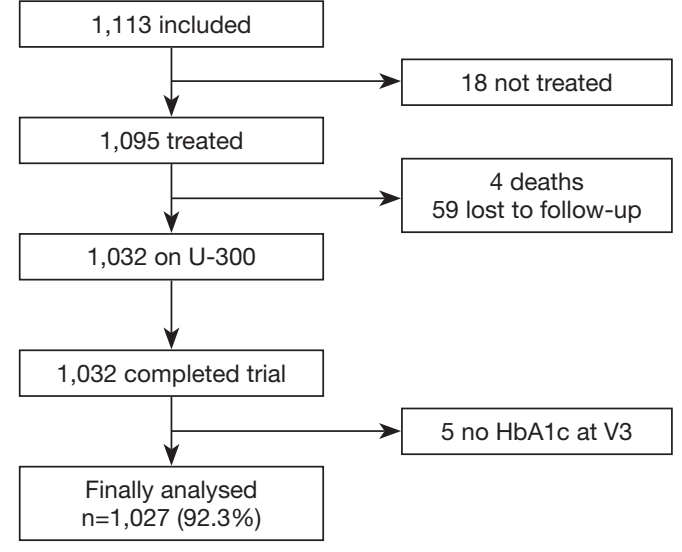

Figure 1 Patient disposition. U-300, insulin glargine U-300; V3, visit 3.

algorithm on final HbA1c was assessed.

All the information was entered into an electronic Case Report File (eCRF) by the prescribing physician.

\section{Safety data collection}

Self-reported hypoglycemic events, adverse events (AEs), serious adverse events (SAEs), acute cardiovascular events, reactions at the injection site, quality/technical issues of Gla-300 were obtained from the medical records of the patients by the treating physician. The following types of hypoglycemic events were recorded: any hypoglycemic event (symptomatic or confirmed); any confirmed symptomatic hypoglycemic event (blood glucose $\leq 70 \mathrm{mg} / \mathrm{dL}$ ); any nocturnal hypoglycemic event (during sleep) and any severe hypoglycemic event (an event which requires the assistance of another person if the patient cannot help himself/herself).

During/after each visit, the treating physician entered the data listed above in the eCRF.

\section{Statistical analyses}

Sample size was calculated initially at 1,100 subjects from approximately 130 study centers with $10 \pm 5$ subjects/center. Effectiveness endpoints were assessed in subjects having at least baseline and 6 months HbAlc assessment (analyzable population-per protocol population). No method for imputing missing data was used. Safety was assessed on the safety population set, defined as all subjects included in the trial that received at least one dose of Gla-300. Safety assessments included self-reported AEs and self-reported hypoglycemic episodes.

Descriptive statistical analysis was performed. In addition, for the change in trial outcomes statistical testing included the $95 \% \mathrm{CI}$ : and used a paired samples $\mathrm{T}$ test to evaluate if the change registered from baseline was statistically significant. Testing was performed at a significance level of $a=0.05$. The SPSS v21 software was used for statistical analysis.

\section{Results}

\section{Patient population}

A total of 1,113 T2DM subjects were included in 124 study sites. Of these, 1,095 received at least one dose of Gla300 and represent the safety population. According to the patient disposition (Figure 1), the population attending V3 with an available $\mathrm{HbA1} \mathrm{c}$ at this timepoint included 1,027 subjects-per protocol population that was finally analyzed.

Baseline clinical and demographic characteristics are reported in Table 1 and are typical for a population with long standing T2DM (mean duration of 8.8 years) requiring treatment intensification with a basal insulin. The study population had a balanced gender distribution, mean BMI of $31.7 \mathrm{~kg} / \mathrm{m}^{2}$ and not at glycemic target with a mean HbA1c of $9.8 \%(83.6 \mathrm{mmol} / \mathrm{mol})$. Majority of subjects were taking metformin $(\sim 88 \%)$ and/or a sulphonylurea $(\sim 75 \%)$. Most subjects presented cardiovascular risks factors, including hypertension (76.1\%), dyslipidemia (72.0\%) and obesity $(70.5 \%)$. We also found that 138 subjects $(13.4 \%)$ were active smokers. The frequency of chronic microvascular and macrovascular diabetes complications is reported in Table 1 .

\section{Efficacy}

\section{Glycemic endpoints}

Treatment with Gla-300 led to a robust decrease of HbA1c in the per protocol population both at 3 months (data available for 542 subjects) - mean \pm SD change of $-1.9 \% \pm 1.9 \%(95 \% \mathrm{CI}:-2.1 \%,-1.7 \%, \mathrm{P}<0.001)$ and 6 months (data available for all 1,027 subjects) $-2.1 \% \pm 2 \%$ (95\% CI: $-2.2 \%,-2.0 \%, \mathrm{P}<0.001$ ) timepoints (Table 2). There was no correlation between the titration algorithm used by investigators and the change in $\mathrm{HbAlc}$ from baseline to the end of trial.

Overall, 383 subjects (37.3\%, 95\% CI: 34.4\%, 40.3\%) reached the HbA1c target of $7 \%$ after a mean duration of insulin Gla-300 treatment of $18.2 \pm 8$ weeks. The proportion was higher in subjects with a lower HbA1c at baseline $(<8 \%)$ 
Table 1 Baseline demographic and clinical characteristics (per protocol analysis, $\mathrm{n}=1,027$ )

\begin{tabular}{|c|c|}
\hline Characteristic & Values \\
\hline Age (years), mean \pm SD (range) & $61.1 \pm 8.5$ (30 to 80$)$ \\
\hline Male/female, n (\%) & $460(44.8) / 567(55.2)$ \\
\hline Weight $(\mathrm{kg})$, mean \pm SD & $88.0 \pm 16.9$ \\
\hline BMI $\left(\mathrm{kg} / \mathrm{m}^{2}\right)$, mean $\pm \mathrm{SD}$ & $31.7 \pm 5.4$ \\
\hline \multicolumn{2}{|l|}{ BMI group, n (\%) } \\
\hline$<25 \mathrm{~kg} / \mathrm{m}^{2}$ & $90(8.8)$ \\
\hline$\geq 25$ to $<30 \mathrm{~kg} / \mathrm{m}^{2}$ & $330(32.1)$ \\
\hline$\geq 30 \mathrm{~kg} / \mathrm{m}^{2}$ & $606(59.1)$ \\
\hline Diabetes duration (years), mean $\pm S D$ & $8.8 \pm 5.2$ \\
\hline $\mathrm{HbA1c}(\%)$, mean $\pm \mathrm{SD}$ & $9.85 \pm 1.7$ \\
\hline \multicolumn{2}{|l|}{ HbA1c group, n (\%) } \\
\hline$<7.0 \%$ & $4(0.4)$ \\
\hline$\geq 7.0 \%$ to $<8.0 \%$ & $79(7.7)$ \\
\hline$\geq 8.0 \%$ & $944(91.9)$ \\
\hline $\mathrm{FPG}(\mathrm{mg} / \mathrm{dL})$, mean $\pm \mathrm{SD}$ & $231.5 \pm 67.4$ \\
\hline \multicolumn{2}{|c|}{ Diabetes complications/comorbidities, n (\%) } \\
\hline Neuropathy & $515(50.1)$ \\
\hline Retinopathy & $107(10.4)$ \\
\hline Chronic kidney disease & $116(11.3)$ \\
\hline Myocardial infarction/stroke & $40(3.9) / 58(5.6)$ \\
\hline Heart failure & $81(7.9)$ \\
\hline Peripheral artery disease & $101(9.8)$ \\
\hline Hypertension & $782(76.1)$ \\
\hline Dyslipidemia & $739(72.0)$ \\
\hline \multicolumn{2}{|l|}{ Diabetes treatment at baseline, $\mathrm{n}(\%)$} \\
\hline Metformin & $908(88.4)$ \\
\hline Sulphonylureas & $774(75.4)$ \\
\hline GLP-1 RAs & $82(8.0)$ \\
\hline DPP-4 inhibitors & $173(16.8)$ \\
\hline Meglitinides & $23(2.2)$ \\
\hline SGLT-2 inhibitors & $39(3.8)$ \\
\hline$\alpha$-glucosidase inhibitor & $116(11.3)$ \\
\hline Thiazolidinediones & $12(1.2)$ \\
\hline
\end{tabular}

$\mathrm{SD}$, standard deviation; $\mathrm{n}$, number of patients; BMI, body mass index; HbA1c, glycated hemoglobin; FPG, fasting plasma glucose; GLP-1 RAs, glucagon like peptide 1 receptor agonists; DPP-4, dipeptidyl peptidase 4; SGLT-2, sodium glucose cotransporter-2.
[45.8\% (95\% CI: $35.5 \%, 56.4 \%)]$ compared to those with a higher HbA1c at baseline ( $\geq 8 \%$ ) [29.3\% (95\% CI: 26.5\%, $32.3 \%)$ ].

Improvement of $\mathrm{HbA1c}$ was accompanied by a significant decrease of the fasting plasma glucose (FPG) values (Table 2). Thus, from a baseline of $231.5 \pm 67.4 \mathrm{mg} / \mathrm{dL}$ (95\% CI: $227.1-235.9 \mathrm{mg} / \mathrm{dL}$ ), data available for 885 subjects, mean change recorded after 3 months (data available for 876 subjects) was $-87.5 \pm 71.4 \mathrm{mg} / \mathrm{dL}$ (95\% CI: -92.2 , $-82.8 \mathrm{mg} / \mathrm{dL}, \mathrm{P}<0.001)$ and plateaued at 6 months (data available for 880 subjects) with a mean change of $-94.8 \pm 72.5$ (95\% CI: $-99.6,-90.0, \mathrm{P}<0.001)$. Final mean FPG was $136.8 \pm 33.7 \mathrm{mg} / \mathrm{dL}$ (95\% CI: $134.6-139.0 \mathrm{mg} / \mathrm{dL}$ ), above recommended targets, indicating insufficient titration of insulin.

\section{Change in body weight}

The mean \pm SD change in body weight from baseline (data available for 1,026 subjects) to 3 months (data available for 1,025 subjects) was of $0.1 \pm 3.2 \mathrm{~kg}(95 \% \mathrm{CI}:-0.1,0.3)$ and of $0.4 \pm 4.5 \mathrm{~kg}$ (95\% CI: $0.1-0.7, \mathrm{P}=0.01)$ at 6 months (data available for all 1,027 subjects). Despite being marginally statistically significant, weight gain of $0.4 \mathrm{~kg}$ after 6 months of treatment with insulin Gla-300 was clinically not relevant, especially taken into account the marked improvement of glycemic control.

\section{Change of insulin dose}

Mean insulin dose recommended at Gla-300 treatment initiation was of $0.26 \pm 0.2 \mathrm{IU} / \mathrm{kg}$ (95\% CI: $0.25-0.27$ ), in accordance with American Association of Clinical Endocrinologists (AACE) guideline recommending a $0.2-0.3 \mathrm{IU} / \mathrm{kg}$ dose if $\mathrm{HbAlc}$ is above $8 \%$ (18). Titration of insulin after baseline led to a mean insulin dose increase of $0.1 \pm 0.2 \mathrm{IU} / \mathrm{kg}$ (95\% CI: $0.09-0.11)$ at 3 months and of $0.2 \pm 0.3 \mathrm{IU} / \mathrm{kg}$ (95\% CI: 0.18-0.22) at 6 months. Final insulin Gla-300 dose at 6 months reached $0.39 \pm 0.2 \mathrm{IU} / \mathrm{kg}(95 \% \mathrm{CI}$ : $0.38-0.40)$. In the majority of cases $(85.3 \%)$ investigators declared basal insulin titration will continue after study completion. In 64 cases (6.2\%), subjects received "rescue" therapy with prandial insulin from baseline to visit V3.

\section{Titration algorithm and correlation with final HbA1c}

Titration was self-performed by the patient according to the titration algorithm agreed with the prescribing physician in $89.1 \%$ of cases at visit V2 and by $90.3 \%$ of subjects at visit V3. Overall, $28.1 \%$ of subjects used a titration algorithm with $2 \mathrm{IU}$ per week while $65.5 \%$ of subjects used a titration 
Table 2 Glycemic endpoints (per protocol analysis, $\mathrm{n}=1,027$ )

\begin{tabular}{lccr}
\hline Characteristic & Baseline & 3 months & 6 months \\
\hline HbA1c, $n$ & 1,027 & 542 & 1,027 \\
HbA1c $(\%)$, mean \pm SD & $9.8 \pm 1.7$ & $8.0 \pm 1.2$ & $7.7 \pm 1.2$ \\
HbA1c change, mean \pm SD $(95 \%$ Cl) & - & $-1.9 \pm 1.9(-2.1,-1.7) ; P<0.001$ & $-2.1 \pm 2.0(-2.2,-2.0) ; P<0.001$ \\
FPG, n & 885 & 876 & 880 \\
FPG (mg/dL) & $231.5 \pm 67.4$ & $144.1 \pm 36.3$ & $136.8 \pm 33.7$ \\
FPG change, mean \pm SD $(95 \%$ Cl) & - & $-87.5 \pm 71.4(-92.2,-82.8) ; P<0.001$ & $-94.8 \pm 72.5(-99.6,-90.0) ; P<0.001$ \\
\hline
\end{tabular}

HbA1c, glycated hemoglobin; n, number of patients; FPG, fasting plasma glucose; SD, standard deviation; Cl, confidence interval.

Table 3 Hypoglycemic events (safety population, $n=1,095$ )

\begin{tabular}{|c|c|c|}
\hline Characteristic & 3 months $\left(n=1,069^{\star}\right)$ & 6 months $\left(n=1,069^{\star}\right)$ \\
\hline Confirmed hypoglycemia ( $\leq 70$ mg/dL), n (\%) & $15(1.4)$ & $28(2.6)$ \\
\hline Nocturnal hypoglycemia, n (\%) & $0(0.0)$ & $7(0.7)$ \\
\hline Severe hypoglycemia, n (\%) & $0(0.0)$ & $1(0.1)$ \\
\hline
\end{tabular}

*, for 26 subjects the eCRF lacked data regarding occurrence of hypoglycemic episodes. $n$, number of patients.

algorithm with 2-3 IU every 3-4 days.

There was no difference between the final achieved HbA1c at 6 months and the titration algorithm used by the subjects. Thus, final HbA1c was $7.7 \% \pm 1.2 \%$ (95\% CI: 7.6-7.8\%) in subjects using a titration algorithm with $2 \mathrm{IU}$ every $3-4$ days, $7.7 \% \pm 1 \%$ (95\% CI: 7.6-7.8\%) in subjects using a titration algorithm with 3 IU every $3-4$ days and $7.9 \% \pm 1.4 \%$ (95\% CI: $7.6-8.1 \%)$ in subjects using a titration algorithm with $2 \mathrm{IU}$ every week.

\section{Safety}

Treatment with insulin Gla-300 was well tolerated. Overall, 126 subjects (11.5\%, 95\% CI: 9.7-13.5\%) reported a total of 264 AEs during the trial. Of these, 25 subjects $(2.3 \%, 95 \%$ CI: $1.6-3.3 \%)$ reported a SAE. Among these, there were 4 deaths (evaluated by investigators not to be related with the trial product) and one case of intentional overdosing of insulin Gla-300 that resulted in a symptomatic hypoglycemic event. There was only one case $(0.1 \%)$ of a technical complaint regarding the injecting device and only one case $(0.1 \%)$ of local reaction at the injection site.

Frequency of hypoglycemic events during Gla300 treatment was low, with a total rate of confirmed $(\leq 70 \mathrm{mg} / \mathrm{dL})$ hypoglycemic events of 0.18 per person per year (PPPY). There were 7 cases of nocturnal hypoglycemic events ( $0.7 \%$ of subjects) with a rate of 0.03 events PPPY. There was only one case of severe hypoglycemia $(0.1 \%$ of subjects). A more detailed description of hypoglycemic events recorded during the trial is given in Table 3.

\section{Discussion}

To test the effectiveness and safety of insulin Gla-300 in real life, we performed an RWE observational/noninterventional trial in a large cohort of 1,095 insulin-naïve T2DM subjects. After 6 months of treatment, starting from a baseline HbA1c of $9.8 \% \pm 1.7 \%$, we recorded a mean decrease of $\mathrm{HbA1c}$ of $2.1 \% \pm 2 \%$. HbA1c reductions recorded in this study are comparable with those obtained in other RWE studies with Gla-300. For instance, in the DELIVER Naïve D study (comparing Gla-300 with insulin degludec), treatment with Gla-300 in 638 insulin-naive T2D subjects with a mean HbA1c at baseline of $9.67 \%$ led to a mean HbA1c decrease of $1.67 \%$ after 6 months, similar with that recorded for degludec treated subjects (1.58\%) (19). In the DELIVER Naïve study (comparing Gla-300 with Gla-100) (20), treatment with Gla-300 in 1,004 insulin-naïve T2DM subjects with a baseline HbA1c of $9.56 \%$ led to a mean HbA1c decrease of $1.52 \%$ after 
6 months that was numerically greater compared with that recorded in 2,008 Gla-100 treated subjects $(1.3 \%$ HbA1c reduction). The slightly higher decrease of $\mathrm{HbA1c}$ in our trial might be explained by its prospective nature (compared to retrospective Electronic Medical Records design of the DELIVER studies) as well as other differences in baseline characteristics of the trial population. Reductions are also comparable with those recorded in the EDITION-3 randomized controlled trial (21) that included insulinnaïve T2DM subjects, with a mean $\mathrm{HbA1c}$ reduction of $1.42 \% \pm 0.05 \%$, indicating a consistency of Gla- 300 glycemic results in RWEs and RCTs.

Another retrospective observational RWE study from the USA analyzed dosing patterns and glycemic outcomes for T2D subjects initiating or switching to Gla-300 (22). In the cohort of 390 insulin-naive patients, 298 subjects have initiated treatment with Gla-300. After 6 months of treatment, the mean least-square change in HbA1c was $1.21 \%$ from a baseline value of $8.63 \%$. This was obtained with a mean daily dose of $0.44 \mathrm{IU} / \mathrm{kg}$ and with a significantly lower risk of hypoglycemia compared to the Gla-100 group (22). The lower drop of HbA1c in this trial might be explained by the lower baseline HbA1c value. An RWE trial with similar design performed in Canada (The REALITY Study) included an insulin-naïve T2DM cohort of 188 subjects treated with Gla-300 and 188 treated with Gla-100 (23). From a baseline HbA1c of $9.76 \% \pm 1.75 \%$, the mean HbA1c change at 6 months was of $-1.78 \% \pm 1.85 \%$, a result almost identical with that recorded in our study. This was obtained with a mean insulin dose of $0.35 \pm 0.22 \mathrm{IU} / \mathrm{kg}$, compared to $0.39 \pm 0.2 \mathrm{IU} / \mathrm{kg}$ in our trial.

Similar improvements in HbA1c were reported by RWE trials in which T2DM subjects treated with other basal insulins were switched to Gla-300 $(24,25)$.

Regarding the percentage of subjects reaching the target of $\mathrm{HbA} 1 \mathrm{c}$ of $7 \%$, a total of $30.7 \%$ of patients in our trial reached this HbAlc target. In comparison, in the DELIVER Naïve D study, $23.8 \%$ of subjects attained the target HbA1c of 7\% (19) while in the DELIVER Naïve study the percentage was of $25 \%$ (20). In the Canadian REALITY study, $27 \%$ of subjects reached the $7 \%$ HbA1c target (23), again very close to the percentage recorded in the current GOAL_RO trial. In our trial, the percentage of subjects reaching target was higher $(45.5 \%)$ in subjects with a lower HbA1c at baseline, highlighting the importance of an early treatment initiation after failure of non-insulin diabetes treatments.

In our trial, treatment with Gla-300 proved to be safe, with only $4.4 \%$ of subjects reporting an episode of symptomatic or confirmed hypoglycemia and only 1 patient $(0.1 \%)$ with an episode of severe hypoglycemia. The rate of confirmed hypoglycemic events was 0.18 events per person per year (PPPY). For comparison, in the DELIVER Naïve study, the incidence of all hypoglycemic events in the Gla300 treated subjects was $9.7 \%$ (20), with a rate of 0.35 events PPPY. In the DELIVER Naïve D study, the total incidence of hypoglycemic events was $10.3 \%$ for Gla-300 subjects, with an event rate of 0.45 PPPY. Finally, in the REALITY study, the proportion of subjects reporting at least an episode of hypoglycemia was $8.7 \%$. The frequency of hypoglycemic events in our trial was again much lower compared to that recorded in the EDITION-3 trials (21). Differences might be explained by the differences in patient populations, insulin titration algorithms and definition/ collection of hypoglycemic events. We hypothesize that insufficient titration of Gla-300 in our trial, indicative of clinical titration inertia, (insulin dose increased by only $0.2 \pm 0.3 \mathrm{IU} / \mathrm{kg}$ over 6 months despite final FPG value at $136 \mathrm{mg} / \mathrm{dL}$ being above the $80-100 \mathrm{mg} / \mathrm{dL}$ target included in the protocol) might have also contributed to the lower frequency of hypoglycemia in our cohort compared to similar RWE trials and, especially to RCTs.

Our study has several limitations. First, this is a nonrandomized, real life, observational, trial with all the inherent drawbacks compared to RCTs. Inclusion of subjects was at the latitude of investigators so that a selection bias cannot be ruled out. For instance, the medications used at baseline (with $16.8 \%$ of subjects on DPP $4 \mathrm{i}$ and $8 \%$ on GLP-1 RAs) does not reflect the current pattern from the general T2DM population in Romania. SMBG was not reinforced by the use of a specific journal/log and this might explain also the low frequency of hypoglycemic episodes recorded in our trial. Finally, different outcome values were not available for all subjects both at the 3 months (V2) and 6 months (V3) evaluations. This is why the findings of our study cannot be generalized to the whole insulin-naïve T2DM population in Romania.

Our study has also some strengths, including the large number of subjects (to our best knowledge this is the largest observational study analyzing the practice of Gla-300 insulin initiation in a real-life practice setting in Romania) and its prospective design.

\section{Conclusions}

In this prospective, observational/non-interventional trial 
performed in an insulin-naïve T2DM population we found a significant decrease of HbA1c and FBG after 6 months of treatment with insulin Gla-300. Titration inertia was evidenced, with a mean insulin dose increase of only $15 \mathrm{IU}$ despite a mean FPG above target. There were no unexpected safety signals, with an overall low number of hypoglycemic events and modest/no clinically relevant weight gain.

The results of this prospective real-world evidence trial confirm the effectiveness and safety results obtained with Gla-300 in T2DM subjects in other populations and complement those of Gla-300 from the randomized controlled trials.

\section{Acknowledgments}

Part of the data reported in this manuscript were previously presented at the International Diabetes Federation (IDF) Congress, Busan, South Korea, 2-6 December 2019.

The GOAL_Ro Study Group: Dr. Monica Andrei, Dr. Liliana Angelescu, Dr. Veronica Anghel, Dr. Tatiana Antoneac, Dr. Diana Aron, Dr. Cristina Ioana Avram, Dr. Ramona Ban, Dr. Simona Cristina Berbece, Dr. Marian Burtea, Dr. Ramona Caziuc, Dr. Simona Chirilă, Dr. Nicolița Chitea, Dr. Mădălina Cîrstoiu, Dr. Simona Clus, Dr. Claudiu Cobuz, Dr. Irina Cojocaru, Dr. Anca Coman, Dr. Ciprian Constantin, Dr. Adrian Copcea, Dr. Dana Cosma, Dr. Elena Costea, Dr. Nicoleta David, Dr. Luiza Demian, Dr. Adela Viorica Dincă, Dr. Garofița Domnițeanu, Dr. Rodica Doros, Dr. Oana Durac, Dr. Simona Eigel, Dr. Georgiana Mihaela Enache, Dr. Anda Fetea, Dr. Adriana Filimon, Dr. Mirela Florea, Dr. Ioana Florin, Dr. Codruta Fotescu, Dr. Eva Fulop, Dr. Carmen Galiano, Dr. Beatrice Ghimiș-Coliță, Dr. Denise Giurgiuman, Dr. Nicoleta Glejaru, Dr. Mihaela Gribovschi, Dr. Cristian Guja, Dr. Olimpia Guțu, Dr. Constanta Iacobescu, Dr. Andra-Liana Ianculescu, Dr. Mihaela Iftene, Dr. Oana Gabriela Iosef, Dr. Ildiko Kicsi-Matyus, Dr. Simona Leca, Dr. Gabriella Lorincz, Dr. Reka Marton, Dr. Maria Mateescu, Dr. Raluca Memu, Dr. Suzana Maria Micu, Dr. Geanina Mihu, Dr. Monica Mocanu, Dr. Corina Moldovan, Dr. Livia Maricica Morariu, Dr. Valentina Mirela Neacşu, Dr. Mirela Neamtu, Dr. Georgiana Nicolescu, Dr. Ruxandra Nicosevici, Dr. Adriana Onaca, Dr. Ionica Paraschiv, Dr. Marlena Pascu, Dr. Melinda Pavel, Dr. Cosmin Pena, Dr. Viorela Pintilii, Dr. Florina Pîrvu, Dr. Mihaela Popoviciu, Dr. Ana Pricope, Dr. Camelia Prună, Dr. Camelia Răcăreanu, Dr. Dana Romanescu, Dr. Adriana Saia-Mihalache, Dr. Luana
Sebestyen, Dr. Cleo Gabriela Simionescu, Dr. Minola Strugariu, Dr. Eleonora Stanca, Dr. Cristian Stanciu, Dr. Daniela Stegaru, Dr. Violeta Stolea, Dr. Daniela Străjer, Dr. Gabriela Strâmbu, Dr. Gabriela Șerban, Dr. Michaela Diaconu Șotropa, Dr. Ramona Ștefan, Dr. Mirela Tache, Dr. Maria Timar, Dr. Cristina Toarba, Dr. Andreea Tocan, Dr. Oana Tudose, Dr. Minodora Țăpurică, Dr. Mihaela Ursache, Dr. Luminita Vanghelie, Dr. Flavia Cristina Vasile, Dr. Oana Vereș, Dr. Maria Mihaela Viruzab, Dr. Oana Vlad, Dr. Delia Vladu, Dr. Cristiana Voineag, Dr. Luminița Voinescu, Dr. Mihaela Luminița Voitec, Dr. Monica Vornicu, Dr. Daniela Zaharie.

Funding: This research was funded by Sanofi Romania. The sponsor was involved in the trial protocol development, data collection, data analysis and manuscript approval.

\section{Footnote}

Reporting Checklist: The authors have completed the TREND reporting checklist. Available at http://dx.doi. org/10.21037/atm-20-4533

Data Sharing Statement: Available at http://dx.doi. org/10.21037/atm-20-4533

Conflicts of Interest: All authors have completed the ICMJE uniform disclosure form (available at http://dx.doi. org/10.21037/atm-20-4533). DS received Speaker fees from Astra Zeneca, Boehringer Ingelheim, Eli Lilly, MSD, Novo Nordisk, Servier Pharma and Sanofi, and was an investigator in the Goal_RO trial sponsored by Sanofi Romania. SN received Speaker fees from Astra Zeneca, Berlin Chemie Menarini, Boehringer Ingelheim, Eli Lilly, MSD, Novo Nordisk, Sanofi, and Servier Pharma and was an investigator in the Goal_RO trial sponsored by Sanofi Romania. DV was an investigator in the Goal_RO trial sponsored by Sanofi Romania. OG received Speaker fees from Astra Zeneca, Boehringer Ingelheim, Eli Lilly, Servier Pharma and Sanofi, and was an investigator in the Goal_RO trial sponsored by Sanofi Romania. AO was an investigator in the Goal_RO trial sponsored by Sanofi Romania. Florina Pîrvu received Speaker fees from Astra Zeneca, Boheringer Ingelheim, MSD, Mylan, Novo Nordisk, Servier Pharma and Worwag Pharma and was an investigator in the Goal RO trial sponsored by Sanofi Romania. MM is an employee of Sanofi Romania. CG has served as a consultant for Astra Zeneca, Boehringer Ingelheim, Eli Lilly, Novo Nordisk, and Sanofi; has received a research grant from UEFISCDI 
Romania; and is on Speakers Bureaus for Astra Zeneca, Boehringer Ingelheim, Eli Lilly, Merck KGaA, MSD, Novo Nordisk, Servier Pharma and Sanofi; he was Study Coordinator of Goal_Ro trial sponsored by Sanofi Romania. CG serves as an unpaid editorial board member of Annals of Translational Medicine from Feb 2018 to Jan 2022. The authors have no other conflicts of interest to declare.

Ethical Statement: The authors are accountable for all aspects of the work in ensuring that questions related to the accuracy or integrity of any part of the work are appropriately investigated and resolved. The study was conducted in accordance with the Declaration of Helsinki from 1964, revised in 2013 (available at http://www.wma. net/en/30publications/10policies/b3/\%20index.html) and its later amendments and the ICH-GCP regulations. Approval by institutional ethics committees was obtained for each participating site. All patients provided written informed consent prior to any study related procedure. Data monitoring was provided by the sponsor.

Open Access Statement: This is an Open Access article distributed in accordance with the Creative Commons Attribution-NonCommercial-NoDerivs 4.0 International License (CC BY-NC-ND 4.0), which permits the noncommercial replication and distribution of the article with the strict proviso that no changes or edits are made and the original work is properly cited (including links to both the formal publication through the relevant DOI and the license). See: https://creativecommons.org/licenses/by-nc-nd/4.0/.

\section{References}

1. International Diabetes Federation. IDF Diabetes Atlas, 9th Edition. Available online: http://www.diabetesatlas.org/

2. Bommer C, Sagalova V, Heesemann E, et al. Global economic burden of diabetes in adults: projections from 2015 to 2030. Diabetes Care 2018;41:963-70.

3. Davies MJ, D'Alessio DA, Fradkin J, et al. Management of hyperglycaemia in type 2 diabetes, 2018. A consensus report by the American Diabetes Association (ADA) and the European Association for the Study of Diabetes (EASD). Diabetologia 2018;61:2461-98.

4. American Diabetes Association. Glycemic Targets: Standards of medical care in diabetes - 2020. Diabetes Care 2020;43:S66-76.

5. Mody R, Huang Q, Yu M, et al. Adherence, persistence, glycaemic control and costs among patients with type 2 diabetes initiating dulaglutide compared with liraglutide or exenatide once weekly at 12-month follow-up in a realworld setting in the United States. Diabetes Obes Metab 2019;21:920-29.

6. Edelman SV, Polonsky WH. Type 2 diabetes in the real world: The elusive nature of glycemic control. Diabetes Care 2017;40:1425-32.

7. Inzucchi SE, Bergenstal RM, Buse JB, et al. Management of hyperglycemia in type 2 diabetes: a patient-centered approach: position statement of the American Diabetes Association (ADA) and the European Association for the Study of Diabetes (EASD). Diabetes Care 2012;35:1364-79.

8. Cefalu WT, Rosenstock J, LeRoith D, et al. Insulin's role in diabetes management: after 90 years, still considered the essential "Black Dress". Diabetes Care 2015;38:2200-3.

9. Russell-Jones D, Pouwer F, Khunti K. Identification of barriers to insulin therapy and approaches to overcoming them. Diabetes Obes Metab 2018;20:488-96.

10. Vargas-Uricoechea H. Efficacy and safety of insulin glargine $300 \mathrm{u} / \mathrm{ml}$ versus $100 \mathrm{u} / \mathrm{ml}$ in diabetes mellitus: a comprehensive review of the literature. J Diabetes Res 2018; 2018:2052101.

11. Marso SP, McGuire DK, Zinman B, et al. Efficacy and safety of degludec versus glargine in type 2 diabetes. $\mathrm{N}$ Engl J Med 2017;377:723-32.

12. Chatterjee S, Khunti K, Davies MJ. Achieving glycaemic control with concentrated insulin in patients with type 2 diabetes. Drugs 2019;79:173-86.

13. Steinstraesser A, Schmidt R, Bergmann K, et al. Investigational new insulin glargine $300 \mathrm{U} / \mathrm{ml}$ has the same metabolism as insulin glargine $100 \mathrm{U} / \mathrm{ml}$. Diabetes Obes Metab 2014;16:873-76.

14. Becker RH, Dahmen R, Bergmann K, et al. New insulin glargine 300 Units.mL-1 provides a more even activity profile and prolonged glycemic control at steady state compared with insulin glargine 100 Units.mL-1. Diabetes Care 2015;38:637-43.

15. Ritzel R, Roussel R, Bolli GB, et al. Patient-level metaanalysis of the EDITION 1, 2 and 3 studies: glycaemic control and hypoglycaemia with new insulin glargine 300 $\mathrm{U} / \mathrm{ml}$ versus glargine $100 \mathrm{U} / \mathrm{ml}$ in people with type 2 diabetes. Diabetes Obes Metab 2015;17:859-67.

16. Makady A, de Boer A, Hillege H, et al. What is realworld data? A review of definitions based on literature and stakeholder interviews. Value Health 2017;20:858-65.

17. Seaquist ER, Anderson J, Childs B, et al. Hypoglycemia and diabetes: a report of a workgroup of the American Diabetes Association and the Endocrine Society. Diabetes 
Care 2013;36:1384-95.

18. Garber AJ, Abrahamson MJ, Barzilay JI, et al. Consensus statement by the American Association of Clinical Endocrinologists and American College of Endocrinology on the comprehensive type 2 diabetes management algorithm - 2019 Executive Summary. Endocr Pract 2019;25:69-100.

19. Sullivan SD, Nicholls CJ, Gupta RA, et al. Comparable glycaemic control and hypoglycaemia in adults with type 2 diabetes after initiating insulin glargine 300 units $/ \mathrm{mL}$ or insulin degludec: The DELIVER Naïve D real-world study. Diabetes Obes Metab 2019;21:2123-32.

20. Bailey TS, Zhou FL, Gupta RA, et al. Glycaemic goal attainment and hypoglycaemia outcomes in type 2 diabetes patients initiating insulin glargine $300 \mathrm{units} / \mathrm{mL}$ or 100 units/mL: Real-world results from the DELIVER Naïve cohort study. Diabetes Obes Metab 2019;21:1596-605.

21. Bolli GB, Riddle MC, Bergenstal RM, et al. New insulin glargine $300 \mathrm{U} / \mathrm{ml}$ compared with glargine $100 \mathrm{U} / \mathrm{ml}$ in insulin-naive people with type 2 diabetes on oral glucoselowering drugs: a randomized controlled trial (EDITION

Cite this article as: Stegaru D, Nicodim S, Vladu D, Guțu O, Onaca A, Pîrvu F, Moise M, Guja C; for the GOAL_RO Study Group. Effectiveness and safety of insulin glargine Gla-300 in insulin-naïve type 2 diabetes subjects in a real-life settingthe GOAL_RO trial. Ann Transl Med 2021;9(2):105. doi: 10.21037/atm-20-4533
3). Diabetes Obes Metab 2015;17:386-94.

22. Gupta S, Wang H, Skolnik N, et al. Treatment dosing patterns and clinical outcomes for patients with type 2 diabetes starting or switching to treatment with insulin glargine (300 Units per milliliter) in a real-world setting: A retrospective observational study. Adv Ther 2018;35:43-55.

23. Abitbol A, Brown RE, Jiandani D, et al. Real-world health outcomes of insulin glargine $300 \mathrm{u} / \mathrm{ml}$ vs insulin glargine $100 \mathrm{u} / \mathrm{ml}$ in adults with type 1 and type 2 diabetes in the Canadian LMC diabetes patient registry: The REALITY Study. Can J Diabetes 2019;43:504-9.

24. Wiesli P, Schories M. Improved glycemic control with insulin glargine $300 \mathrm{U} / \mathrm{mL}$ (Toujeo $囚$ ) in patients with type 2 diabetes: real-world effectiveness in Switzerland. Diabetes Ther 2018;9:2325-34.

25. Zhou FL, Ye F, Berhanu P, et al. Real-world evidence concerning clinical and economic outcomes of switching to insulin glargine 300 units/mL vs other basal insulins in patients with type 2 diabetes using basal insulin. Diabetes Obes Metab 2018;20:1293-97. 